\title{
METHOD TO DETERMINE THE ABSORPTANCE OF THIN FILMS FOR PHOTOVOLTAIC TECHNOLOGY*
}

\author{
Nathan A. Tomlin ${ }^{1}$, John H. Lehman ${ }^{1}$, Katherine E. Hurst ${ }^{2}$, David B. Tanner ${ }^{3}$, K. Kamarás ${ }^{4}$, Áron Pekker ${ }^{4}$ \\ ${ }^{1}$ National Institute of Standards and Technology, Optoelectronics Division, Boulder, CO, U.S.A. \\ ${ }^{2}$ National Renewable Energy Laboratory, Chemical and Material Sciences, Golden, CO, U.S.A \\ ${ }^{3}$ Department of Physics, University of Florida, Gainesville, Florida, U.S.A. \\ ${ }^{4}$ Research Institute for Solid State Physics and Optics, Hungarian Academy of Sciences, Budapest, Hungary
}

\begin{abstract}
We have demonstrated a novel method to determine optical properties of opaque or semi-transparent films for photovoltaic (PV) applications. Such films may be the basis of transparent conductors or photoconductive material. As an example, we measure the absolute absorptance (at visible and near infrared wavelengths) of an optically thick single-wall carbon nanotube (SWCNT) film by using a pyroelectric detector. This novel method obviates the need for analysis with respect to polarization and associated difficulties of ellipsometry. The KramersKronig relation is used to determine the thick film index of refraction, which we use to calculate the optical properties of thin films as a function of thickness. A transmittance measurement obtained from a thin SWCNT film shows excellent agreement with results from our model.
\end{abstract}

\section{INTRODUCTION}

Current thin film characterization techniques include photothermal deflection spectroscopy, ellipsometry, and spectrophotometer measurements [1], [2]. We have developed a novel technique whereby we determine the spectral absorptivity of an opaque film by evaluating the film as a detector coating for a pyroelectric thermal detector. From the absolute absorptance and the Kramers-Kronig relation, we determine the index of refraction, which we use to investigate the spectral properties of carbon nanotubes and carbon nanotubebearing films with respect to thickness. Unlike other thin film characterization techniques, our absolute absorptance measurement is polarization independent and provides more spectral information.

\section{EXPERIMENT}

For our absolute absorptance measurement, we required an optically thick film of SWCNTs. We started with SWCNTs synthesized by a laser vaporization method similar to that reported previously [3], [4]. The SWCNTs were purified by oxidation of the raw soot in flowing $\mathrm{CO}_{2}$ at $800{ }^{\circ} \mathrm{C}$ for one hour, followed by a reflux in 3 mola $\mathrm{HNO}_{3}$ for 16 hours. The solution was then filtered, the filtrand was washed, and then dried at $50{ }^{\circ} \mathrm{C}$ for 30 minutes. During this drying process the SWCNTs separated from the filter, producing a free-standing "buckypaper" or film (Fig. 3 inset). The film was further oxidized in air at $550^{\circ} \mathrm{C}$
${ }^{*}$ Contribution of the U.S. Government; not subject to copyright.

for 30 minutes. The resulting material purity was greater than $97 \%$ by weight as determined by thermogravimetric analysis.

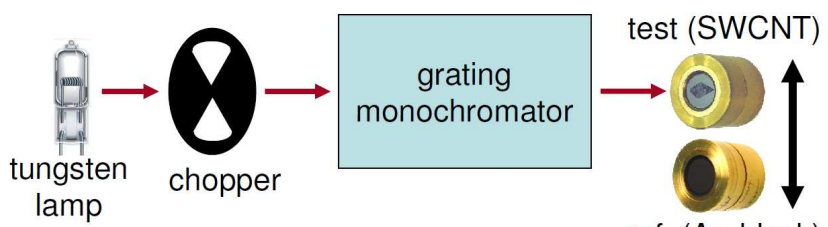

Figure 1 Schematic of the setup used to measure absolute spectral responsivity of the thick SWCNT film.

Our spectral responsivity measurement system consists of a chopped lamp source, grating monochromator, and two pyroelectric detectors, one of which is a NIST transferstandard detector (Fig. 1). Direct substitution provides absolute spectral responsivity relative to the NIST standard at $10 \mathrm{~nm}$ wavelength increments from $600 \mathrm{~nm}$ to $2000 \mathrm{~nm}$. The beam exiting the monochromator is focused on the detector to a beam size of approximately $2 \mathrm{~mm}$ by $2 \mathrm{~mm}$, normal to the plane of the detector surface, with a bandwidth of $6 \mathrm{~nm}$.
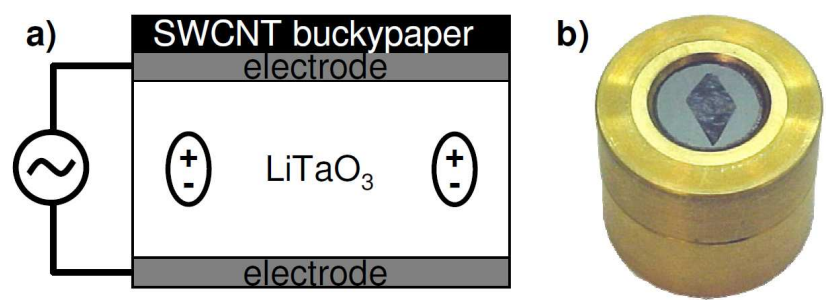

Figure 2 (a) Schematic and (b) picture of the pyroelectric detector with thick SWCNT film attached.

The current generated by a pyroelectric detector is proportional to the volume average of the change in temperature as a function of time. Therefore, the measured spectral responsivity depends only on the conversion of optical energy to thermal energy by the coating. Each pyroelectric detector is prepared from a zcut $\mathrm{LiTaO}_{3}$ plate, $12 \mathrm{~mm}$ in diameter and $60 \mu \mathrm{m}$ thick, with electrodes on both sides (Fig. 2). The back electrode is 10 
$\mathrm{mm}$ in diameter and consists of $50 \mathrm{~nm}$ of gold on top of 25 $\mathrm{nm}$ of chromium. The front electrode is $25 \mathrm{~nm}$ of chromium. The reference transfer-standard detector was fabricated with a gold-black coating on the front electrode, giving it an absorption efficiency greater than $99 \%$ over the measured spectral range [4]. The prepared buckypaper was attached to the front electrode of the test detector using a drop of chloroform [5]. Each pyroelectric detector was connected to the signal input of a current amplifier with $10^{-10} \mathrm{~A} / \mathrm{V}$ gain. The optical input to the detector was modulated at $15 \mathrm{~Hz}$ and measured with a lock-in detection scheme [6].

As a check on our model, we also prepared an additional thin film from a sample of the same buckypaper, which was sonicated using a probe tip, dispersed in chloroform, and sprayed with an airbrush onto a glass cover slip (Fig. 6 inset) [3]. This resulted in a very thin film of SWCNTs, which was measured with a spectrophotometer to obtain the transmission.

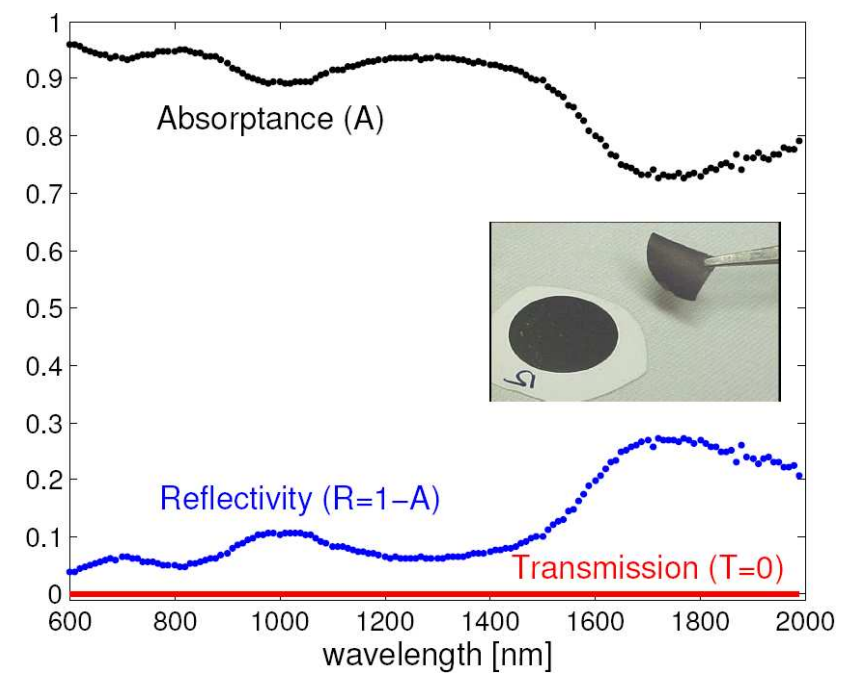

Figure 3 Measured absorptance, calculated reflectivity, and assumed transmission of optically thick SWCNT coating determined from detector responsivity measurement. Inset: Picture of thick SWNCT film (buckypaper).

\section{MODELING}

The thick detector coating is assumed to be optically thick (transmission $T=1$ ), so the absolute responsivity is proportional to absorptance $A$. Since $A+R+T=1$, we can determine reflectivity $R=1-A$ (Fig. 3). The complex reflection coefficient $r(E)$ is

$$
r(E)=\sqrt{R(E)} e^{i \theta(E)}
$$

where $E$ is the photon energy and $\theta$ is the phase of the reflected light. For each datapoint, $R\left(E_{i}\right)$, we can find the corresponding phase at that point, $\theta\left(E_{i}\right)$, using the Kramers-Kronig (KK) relation between $R$ and $\theta$

$$
\theta\left(E_{i}\right)=-\frac{E_{i}}{\pi} \int_{0}^{\infty} \frac{\ln \left(R(E) / R\left(E_{i}\right)\right)}{E^{2}-E_{i}^{2}} d E
$$

Since the Kramers-Kronig relation involves an integral over all energies, and we only have reflectance data for a limited range of energies, we extrapolate our data to lower and higher energies and split the integral in Eq. 2 into three separate integrals

$$
\theta\left(E_{i}\right)=\theta_{\text {low }}\left(E_{i}\right)+\theta_{\text {mid }}\left(E_{i}\right)+\theta_{\text {high }}\left(E_{i}\right)
$$

where $\theta_{\text {low }}$ and $\theta_{\text {high }}$ are integrals over energies outside of the range of measured data. For the low energy integral ( $E<E_{\text {min }}$ ), we assume that $R$ is constant and equal to $R\left(E_{\min }\right)$, which gives

$$
\theta_{\text {low }}\left(E_{i}\right)=-\frac{E_{i}}{\pi} \int_{0}^{E_{\min }} \frac{\ln \left(R\left(E_{\min }\right) / R\left(E_{i}\right)\right)}{E^{2}-E_{i}^{2}} d E
$$

For the middle integral over measured energies ( $E_{\min }<E<E_{\max }$ ), the integral in Eq. 2 blows up for $E=E_{i}$, so we use the MacLauren method to evaluate this integral

$$
\theta_{\text {mid }}\left(E_{i}\right)=-\frac{E_{i}}{\pi} \cdot 2 d E \cdot \sum_{j}^{\prime}\left[\frac{\ln \left(R\left(E_{j}\right) / R\left(E_{i}\right)\right)}{E_{j}^{2}-E_{i}^{2}}\right]
$$

where $d E$ is the interval between energy spacings [7]. The sign $\sum$ denotes that when $i$ is an odd number, then $j=2,4,6, \ldots, i-1, i+1, \ldots$, and when $i$ is an even number, then $j=1,3,5, \ldots, i-1, i+1, \ldots$.

For the high energy integral $\left(E>E_{\max }\right)$, we assume that $R$ falls off as a power law, which we additionally split into two regimes with different power laws,

$$
\begin{aligned}
R\left(E_{\text {max }}<E<E_{\text {free }}\right) & =R\left(E_{\text {max }}\right)\left(E_{\text {max }} / E\right)^{m} \\
R\left(E>E_{\text {free }}\right) & =R_{\text {free }}\left(E_{\text {free } / E}\right)^{m} \text { free }
\end{aligned}
$$

where $E_{\text {free }}$ is the energy past which the free-electron asymptotic limit holds, $m$ is the power law coefficient for $R\left(E_{\max }<E<E_{\text {free }}\right), m_{\text {free }}$ is the power law coefficient for $R\left(E>E_{\text {free }}\right) \quad, \quad$ and $R_{\text {free }}=R\left(E_{\max }\right)\left(E_{\max } / E_{\text {free }}\right)^{m}$ [8]. The high energy integral then becomes

$$
\begin{aligned}
& \theta_{\text {high }}\left(E_{i}\right)= \\
& -\frac{E_{i}}{\pi} \int_{E_{\max }}^{E_{\text {free }}} \frac{\ln \left(\frac{R\left(E_{\max }\right)\left(E_{\max } / E\right)^{m}}{R\left(E_{i}\right)}\right)}{E^{2}-E_{i}^{2}} d E \\
& -\frac{E_{i}}{\pi} \int_{E_{\text {free }}}^{100 \cdot E_{\text {free }}} \frac{\ln \left(\frac{R_{\text {free }}\left(E_{\text {free }} / E\right)^{m} \text { free }}{R\left(E_{i}\right)}\right)}{E^{2}-E_{i}^{2}} d E
\end{aligned}
$$


where we have approximated the limit of infinity on the second integral as $100 \cdot E_{\text {free }}$. For our work, we used values of $m=0.7, m_{\text {free }}=4$, and $E_{\text {free }}=3.2 \cdot 10^{-18} \mathrm{~J}$ $(20 \mathrm{eV})$.

Once we have the complex reflection coefficient $r$, we can use the Fresnel equation for normal incidence

$$
r^{2}=\left(\frac{N_{a i r}-N}{N_{a i r}+N}\right)^{2}
$$

to solve for the complex index of refraction $N$ of the optically thick SWCNT coating

$$
N=n+i k=\frac{1+r}{1-r}
$$

where we have used $N_{\text {air }}=1$ (Fig. 4). We can then calculate the reflection and transmission for a SWCNT film of arbitrary thickness using the transfer matrix method [9]. Fig. 5 shows a plot of calculated transmission for SWCNT thin films of thicknesses from $1 \mathrm{~nm}$ to $1000 \mathrm{~nm}$.

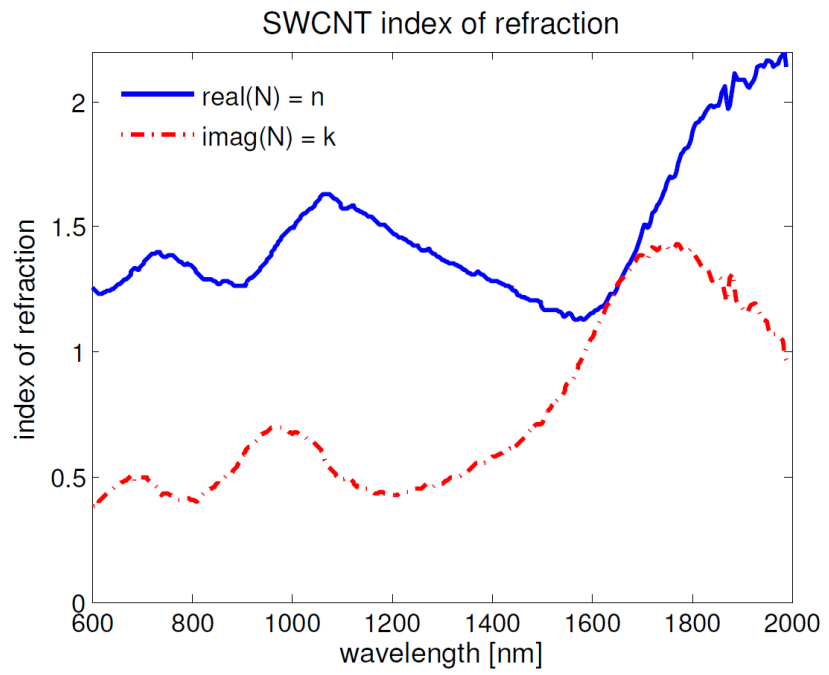

Figure 4 Calculated index of refraction of optically thick SWCNT film determined from Kramers-Kronig relation.

\section{RESULTS}

We have compared our model against a spectrophotometer transmittance measurement of a thin SWCNT film. We found reasonable agreement between theory and measurements, by using a modeled film thickness of $30 \mathrm{~nm}$ as shown in Fig. 6. However, we were unable to obtain a separate measurement of the thin film thickness to compare to the model.

\section{CONCLUSION}

We have demonstrated a novel method to determine optical properties of semi-transparent films. An optically thick coating on a pyroelectric detector allows us to measure the absolute absorptance of the opaque film. The Kramers-Kronig relation provides a means to calculate the index of refraction, which we use to calculate the reflectance and transmittance for thin films of arbitrary thickness. Our calculations show reasonable agreement when compared with measurements on a thin film.

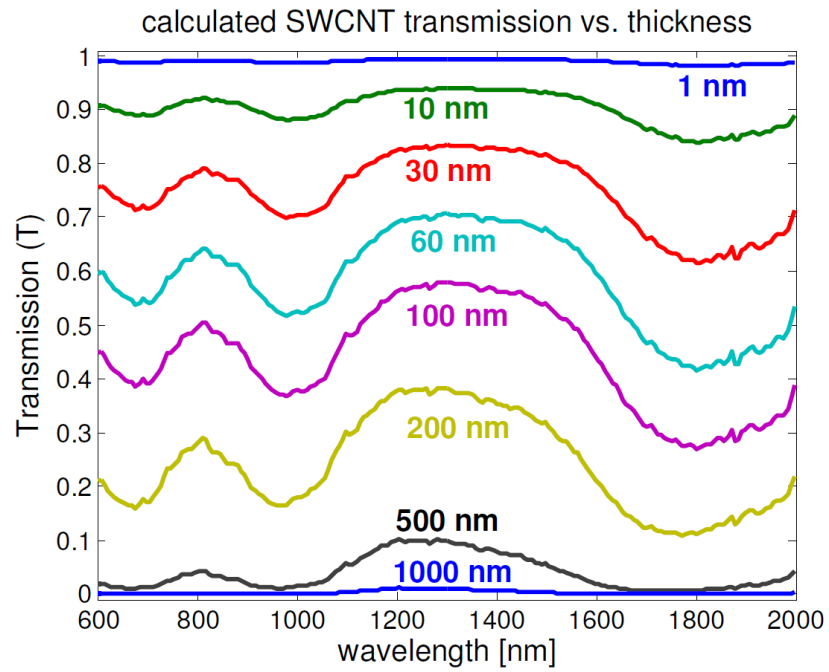

Figure 5 Calculated transmission for SWCNT thin films of varying thickness.

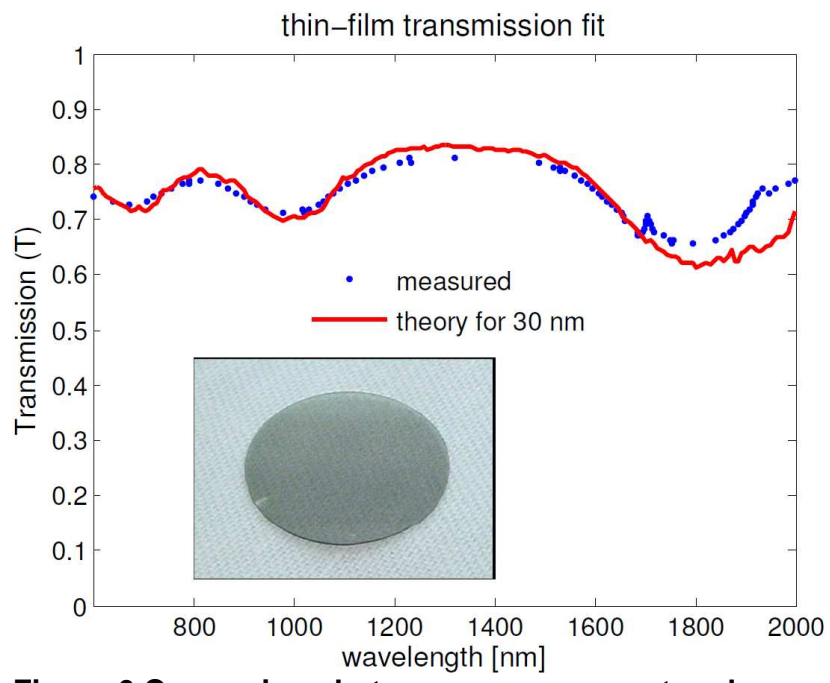

Figure 6 Comparison between measurement and theory for a SWCNT film. Inset: Picture of thin SWCNT film on glass cover slide.

\section{REFERENCES}

[1] W.B. Jackson, N.M. Amer, A.C. Boccara, and D. Fournier, "Photothermal Deflection Spectroscopy and Detection," Appl. Opt. 20, 1981, pp. 1333-1344.

[2] T.M. Barnes, J. Van de Lagemaat, D. Levi, G. Rumbles, T.J. Coutts, C.L. Weeks, D.A. Britz, I. Levitsky, J. Peltola, 
and P. Glatkowski, "Optical Characterization of Highly Conductive Single-Wall Carbon-Nanotube Transparent Electrodes," Phys. Rev. B 75, 2007, p. 235410.

[3] A.C. Dillon, P.A. Parilla, J.L. Alleman, J.D. Perkins, and M.J. Heben, "Controlling Single-Wall Nanotube Diameters with Variation in Laser Pulse Power," Chem. Phys. Lett. 316, 2000, pp. 13-18.

[4] J. Lehman, E. Theocharous, G. Eppeldauer, and C. Pannell, "Gold-Black Coatings for Freestanding Pyroelectric Detectors," Meas. Sci. Tech. 14, 2003, pp. 916-922.

[5] J.H. Lehman, K.E. Hurst, L.K. Roberson, K. Nield, and J.D. Hamlin, "Inverted Spectra of Single-Wall Carbon Nanotube Films," J. Phys. Chem. C 112, 2008, pp. 1177611778.

[6] J. Lehman, G. Eppeldauer, J.A. Aust, and M. Racz, "Domain-Engineered Pyroelectric Radiometer," Appl. Opt. 38, 1999, pp. 7047-7055.

[7] K. Ohta and H. Ishida, "Comparison Among Several Numerical Integration Methods for Kramers-Kronig Transformation," Appl. Spec. 42, 1988, pp. 952-957.

[8] F. Wooten, Optical Properties of Solids, Academic Press, 1972.

[9] G. Fowles, Introduction to Modern Optics, Dover Publications, 1975, pp. 97-99. 